\title{
Arbeitnehmer: Freiwild der Überwachung?
}

\section{Bericht über die Sommerakademie 2009}

Nicht nur in Schleswig-Holstein markieren sich Datenschutzinteressierte jedes Jahr den letzten Montag im August in ihren Kalendern. An diesem Tag findet nämlich traditionell die Sommerakademie des Unabhängigen Landeszentrums für Datenschutz (ULD) in Kiel statt. Dieses Jahr ging es um Beschäftigtendatenschutz. Der Diskussionsbedarf zu diesem Thema zeigte sich schon im Vorfeld am Rekord von mehr als 600 Anmeldungen. Offensichtlich hatten die Skandale der vorangegangenen Monate und die Debatten um ein Arbeitnehmerdatenschutzgesetz das Interesse angefacht. So fühlten sich nicht nur behördliche und betriebliche Datenschutzbeauftragte sowie Vertreter aus Datenschutzbehörden angesprochen, sondern verstärkt auch Personalund Betriebsräte.

\section{Reden und Ergebnisse}

Bereits im Grußwort des Präsidenten des Schleswig-Holsteinischen Landtags Martin Kayenburg kristallisierte sich ein erstes Ergebnis aus: Die Identifikation der Mitarbeiter und Mitarbeiterinnen mit ihrem Unternehmen ist wichtig für den Schutz von Unternehmensdaten. Dagegen sei „ein quasi institutionalisiertes Misstrauen von Vorgesetzten kontraproduktiv“. Kayenburg konstatierte ebenso wie der Leiter des ULD Thilo Weichert einen Regelungsbedarf auf Bundesebene zum Schutz der Beschäftigten in der Privatwirtschaft. Weichert erläuterte dann die (rechtskonforme) Entstehung des Bildes, mit dem das ULD auf die Veranstaltung hinwies: Die plakativ als „FREIWILD“ abgestempelte Arbeitnehmerin, deren Gesichtsfoto sich nun in biometrisch abgleichbarer Qualität in vielfacher Kopie im Internet befindet, gibt es gar nicht. Tatsächlich wurden fünf Fotos von Mitarbeiterinnen (alles auf rein freiwilliger Basis der Mitwirkenden, versteht sich) digital übereinandergelegt, wodurch per Morphing diese hübsche Kunstfigur entstand.

Karin Schuler von der Deutschen Vereinigung für Datenschutz (DVD) berichtete aus ihrer Praxis als Sachverständige für Personalvertretungen und stellte anschaulich dar, woran der Patient „Arbeitnehmerdatenschutz" krankt. Sie rechnete u.a. vor, dass in Deutschland unter der (zu hoch gegriffenen)
Annahme, dass eine Aufsichtsbehörde pro Jahr im Schnitt ca. 1.000 Firmen kontrolliert, ein Unternehmen statistisch gesehen nur alle 218 Jahre mit einer Datenschutzprüfung rechnen müsse. Bettina Gayk von der Landesbeauftragten für Datenschutz und Informationsfreiheit Nordrhein-Westfalen konkretisierte die Zahlen für den Punkt „Beschäftigtendatenschutz“: In ihrem Bundesland stünde dafür die Arbeitskraft von lediglich 2 bis 2,5 Personen den etwa 700.000 nordrhein-westfälischen Unternehmen und den Behörden gegenüber.

Die ehemalige Justizministerin Herta Däubler-Gmelin, die an der Aufklärung von Mitarbeiterbespitzelungen bei mehreren großen Konzernen mitgewirkt hat, plauderte aus dem Nähkästchen: Häufig würde sich das Management nicht selbst die Hände schmutzig machen, sondern andere Firmen für die Überwachung einschalten teilweise auf Basis mündlicher oder diffuser vertraglicher Regelungen. Bei der Rede von Roland Wolf, Bundesvereinigung der Deutschen Arbeitgeberverbände, konnte Däubler-Gmelin nicht nachvollziehen, auf welcher Basis er dem Arbeitnehmerdatenschutz den Grundrechtscharakter absprach. Für Unruhe im Auditorium sorgte Wolfs Vorschlag, bei Überwachungen von Beschäftigten die Zustimmungsnotwendigkeit des Betriebsrats partiell durch eine nachträgliche Informationspflicht zu ersetzen, um etwaigen Interessenkollisionen zu begegnen. Wolf konnte sich nicht den Vorrednern anschließen, die den neuen $\$ 32$ BDSG zum Beschäftigtendatenschutz als zu dünn kritisiert hatten. Einigkeit bestand lediglich in der Frage, dass ein Mehr an rechtlicher Klarheit zu fordern ist.

Martina Perreng vom Deutschen Gewerkschaftsbund (DGB) hatte eine ganze Liste von Verbesserungswünschen, die Beschäftigte insbesondere vor "freiwilligen“ Zugeständnissen schützen sollen, die Arbeitgeber einforderten. Marit Hansen zeigte schließlich die technischen Möglichkeiten der Überwachung auf und machte deutlich, dass Industriespionage und Mitarbeiterüberwachung eng verwandt sein können. Hier sei es nötig, dass Arbeitgeber ihre Beschäftigten zum Schutz vor Ausspähung der persönlichen sowie der Unternehmensdaten befähigten.

Nach dem Mittagessen konnten die Teilnehmenden zweimal zwischen je fünf parallelen Infobörsen wählen, in denen spezi-

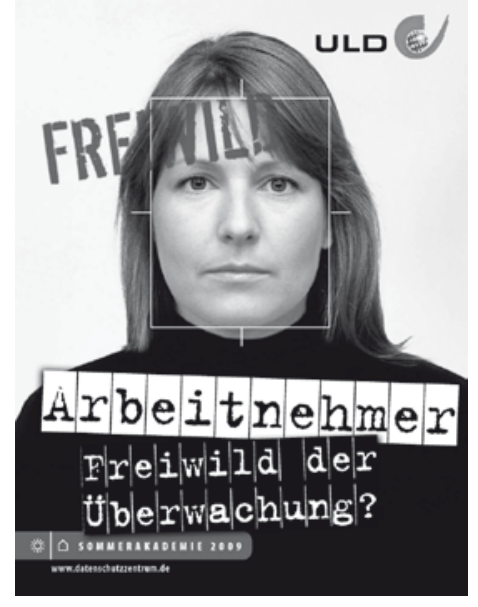

fische Aspekte vertieft diskutiert wurden, u.a. automatisierte Personalverfahren, Bürokommunikationsüberwachung, Filtertechnik bei Internetnutzung, Gesundheitsdatenverarbeitung, internationaler Datenaustausch, Korruptionsbekämpfung, Lokalisierungsdienste, Mitarbeiteraußendarstellung, Verhaltensprotokollierung und Whistleblowing.

Bei der abschließenden Podiumsdiskussion sorgte der zuständige Staatssekretär im Bundesarbeitsministerium Detlef Scheele für eine Überraschung, als er für die nächsten Tage den Entwurf eines Beschäftigtendatenschutzgesetzes ankündigte. Dieser hätte allerdings keine Chance auf Verabschiedung innerhalb der endenden Legislaturperiode.

Lokalkolorit erhielt die Veranstaltung durch die plattdeutsche Laudatio zum Datenschutzaudit für die Amtsverwaltung Viöl in Nordfriesland: Die Amtsgemeinde habe nachgewiesen, „dat all dat Gedöns för Datenschutz un Datensekerheit ok för een lütt Behörd funktschoneern kun, so wie dat akkuraat is un sich de Datenschützers dat wünschen."

\section{Zum Vormerken}

Die nächste Sommerakademie des ULD findet am 30. August 2010 statt. Arbeitstitel: „Codex digitalis universalis - Datenschutz durch neue Normen und Techniken“. Weitere Informationen einschließlich der Skripten und Präsentationen der vergangenen Sommerakademien sind zu finden unter: https://www. datenschutzzentrum.de/sommerakademie/

Marit Hansen, ULD Schleswig-Holstein 